\title{
Perfil clínico de los pacientes ingresados al programa EPOC en un consultorio de atención primaria durante 10 años
}

\author{
GONZALO ALVEAR T.*, LIDIA P. FIGUEROA R.** y CARLOS A. PEÑA M.***
}

\section{Clinical profile of patients admitted to a COPD program in a primary care setting during 10 years}

Our objective was to determine the clinical profile of patients admitted to the COPD program of CESFAM Silva Henriquez of Puente Alto, Chile, between 2004 and 2014. We analyzed the clinical and spirometric data of medical and controls records as being admitted to the program. The predicted FEV $V_{1}$ percentage average of the 65 patients analyzed was $76.6 \% .47 .7 \%$ had mild, $40 \%$ moderate and $12.3 \%$ severe COPD. The patients were 62 years-old in average, 55.4\% were women, 78.5\% had smoking history, 73.6\% had been exposed to wood and/or coal combustion (50.1\% to both), $61.5 \%$ had some comorbidity (90\% cardiovascular). $40 \%$ were under monotherapy with short-acting bronchodilator $(B D c)$ and $50.7 \%$ received dual therapy with BDc and inhaled corticosteroid. Our COPD patients were predominantly women over 60 years old, exposed to tobacco and biomass and most of them had cardiovascular comorbidities.

Key words: COPD; Clinical Profile; Primary care setting.

\section{Resumen}

Nuestro objetivo fue conocer el perfil clínico de los pacientes ingresados al programa EPOC del CESFAM Silva Henríquez de la comuna de Puente Alto entre 2004 y 2014. Para eso analizamos los datos clínicos y espirométricos de las fichas clínicas y cartolas de controles al momento del ingreso al programa. El porcentaje del $\mathrm{VEF}_{1}$ predicho promedio de los 65 pacientes analizados fue de 76,6\%. El 47,7\% fue EPOC leve, 40\% moderado y 12,3\% severo. La edad promedio fue 62 años, el 55,4\% fueron mujeres, 78,5\% tenía antecedentes tabáquicos, 73,6\% había estado expuesto a combustión de leña y/o carbón (50,1\% a ambos), 61,5\% tenía alguna comorbilidad (90\% cardiovascular). Al 40\% se les indicó monoterapia con broncodilatador de acción corta (BDc) y al 50,7\% terapia dual con BDc más corticoide inhalado. Nuestros EPOC fueron predominantemente mujeres mayores de 60 años, expuestas a tabaco y biomasa y con comorbilidades, preferentemente cardiovasculares.

Palabras clave: EPOC; Perfil clínico; Atención primaria; Salas ERA.

\section{Introducción}

La enfermedad pulmonar obstructiva crónica (EPOC) es un importante problema de salud pública ya que es causante de invalidez, contribuye enormemente a la pérdida de años de vida potenciales y ya llegó a ser la $3^{\mathrm{a}}$ causa de muerte en el mundo el año 2010¹. El estudio PLATINO, realizado en países de Sudamérica y México, mostró una prevalencia en Chile del $15,9 \%{ }^{2}$.

Sabemos que la EPOC es una enfermedad extremadamente heterogénea tanto en los síntomas como en el deterioro de la función pulmonar en el tiempo, respuesta a las terapias disponibles y evolución clínico-funcional ${ }^{3}$. Probablemente esta heterogenicidad podría explicarse en gran

\footnotetext{
* Clínica Miguel de Servet e Integramédica. Providencia. Región Metropolitana.

** CESFAM Cardenal Raúl Silva Henríquez. Puente Alto. Región Metropolitana.

***Departamento de Enfermedades Respiratorias, Servicio de Medicina. Hospital San Borja Arriarán. Santiago. Región Metropolitana, Chile.
} 
parte por la interacción entre la susceptibilidad genética individual con la exposición a diferentes tipos y cantidad de factores de riesgo respiratorio y diferencias regionales geográficas ${ }^{4-6}$, incluso dentro de un mismo país ${ }^{7}$. De ahí la importancia de conocer el perfil clínico regional de los pacientes con EPOC ya que existen escasos estudios latinoamericanos que aborden las características clínicas de los pacientes con EPOC $^{6,8} \mathrm{y}$, hasta donde conocemos, ninguno chileno.

Otro aspecto importante es que la mayoría de los estudios se han realizado con estrictos criterios de inclusión y exclusión y muy pocos en el contexto de "vida real", por lo que también se hace necesario conocer a los pacientes en el contexto de "vida real"10, ya que así podremos establecer, entre otras cosas, la severidad y necesidades terapéuticas reales como también proyectar las futuras necesidades y ajustes del manejo programático.

Con esta idea en mente diseñamos este estudio para conocer el perfil clínico de los pacientes EPOC en un centro de salud familiar (CESFAM) de la comuna de Puente Alto en Santiago.

La comuna de Puente Alto tiene 8 centros de atención primaria de salud implementados con sala ERA, de los cuales 6 son CESFAM. Las salas ERA son una estrategia de salud diseñada centralmente para enfrentar el problema diagnóstico y el manejo de algunas patologías respiratorias como la EPOC ${ }^{11}$. Estas salas se instalaron a través de un programa piloto en la Región Metropolitana el año 2000 y actualmente se encuentran extendidas a todas las comunas del país. Las salas ERA están constituidas por un equipo de profesionales (médico, kinesiólogo y enfermera) de la Atención primaria de salud que han sido capacitados por el Ministerio de Salud de Chile y que disponen de protocolos de manejo estandarizado $^{12}$ y mantiene registros propios de sus pacientes. Además, Chile cuenta con una modalidad de garantía de salud (AUGE) para diferentes patologías entre las que se encuentra la EPOC, lo que garantiza disponer, entre otras cosas, de los fármacos inhalados ${ }^{13}$.

La sala ERA del CESFAM Cardenal Raúl Silva Henríquez de Puente Alto, que tiene asignada una población de adultos (mayores de 20 años) de 23.292, funciona desde el año 2004 y actualmente está compuesta por un médico general y dos kinesiólogas.

El objetivo principal de este estudio fue caracterizar a los pacientes EPOC bajo control en la sala ERA del CESFAM Cardenal Raúl Silva Henríquez al momento del ingreso y que se man- tienen con controles activos, según exposición a factores de riesgo respiratorio, presencia y tipo de comorbilidades, deterioro de la función pulmonar y terapia prescrita.

\section{Pacientes y Método}

Este estudio descriptivo retrospectivo no intervencional analizó los datos clínicos y espirométricos de la ficha clínica y cartola de control al momento del ingreso de todos los pacientes controlados por EPOC en la sala ERA desde el año 2004 a junio de 2014 del CESFAM Cardenal Raúl Silva Henríquez de la comuna de Puente Alto.

En todos los pacientes se verificó su inscripción en el sistema estadístico (SIGGES) para garantizar su manejo en el sistema AUGE.

Se analizaron los datos de los pacientes que cumplieron con los siguientes criterios de inclusión: 1) alteración espirométrica compatible con la definición funcional de EPOC según las guías clínicas ministeriales ${ }^{12}$; 2) que la espirometría estuviese disponible para poder analizar si cumplía con los criterios de la SER ${ }^{14}$; 3 ) que el paciente hubiese acudido al menos a un control en la sala ERA durante los últimos 12 meses (pacientes activos).

Los datos analizados fueron edad, género, índice de masa corporal (IMC), consumo de tabaco, exposición a combustión de biomasa, radiografía de tórax, función pulmonar, presencia y tipo de comorbilidades, fármacos indicados, número de exacerbaciones y hospitalizaciones por la enfermedad durante el año previo al ingreso.

El IMC se agrupó en bajo peso $\left(<20 \mathrm{~kg} / \mathrm{m}^{2}\right)$, peso normal $\left(20-24,9 \mathrm{~kg} / \mathrm{m}^{2}\right)$, sobrepeso $(25-29,9$ $\left.\mathrm{kg} / \mathrm{m}^{2}\right)$ y obeso $\left(\geq 30 \mathrm{~kg} / \mathrm{m}^{2}\right)^{15}$. El consumo de tabaco se calculó según el índice paquetes/año. Los resultados de las radiografías de tórax fueron extraídos de los informes radiológicos hechos por diversos radiólogos, por lo que no siempre se dispuso de las imágenes. En los casos que se dispuso de ellas, fueron interpretadas por uno de los autores. La función pulmonar se obtuvo mediante espirometrías realizadas por el kinesiólogo (a) de la sala ERA o por el tecnólogo médico del hospital base. Todas fueron hechas siguiendo las recomendaciones de la SER y con los valores de referencia de Knudson et al. La interpretación de la severidad de la obstrucción se hizo según criterios GOLD $2011^{16}$.

Los resultados son presentados de una manera descriptiva y las variables analizadas en números absolutos, promedios o porcentajes. 


\section{Resultados}

De los 233 pacientes ingresados por EPOC en la sala ERA desde el 2004, 104 habían acudido a control durante el último año. De ellos, 83 tenían espirometría al momento del ingreso a la sala ERA. En los 21 restantes, 7 no tenían registro de realización de la espirometría, 13 tenían registrado sólo el resultado numérico y uno tenía el registro pero no los datos.

De los 83 pacientes con espirometría al ingreso, 18 no cumplían los criterios de EPOC. Diecisiete de ellos fueron ingresados como EPOC en etapa 0. Los datos de los 65 pacientes restantes que cumplieron los criterios espirométricos de EPOC fueron los que se analizaron en el estudio (Figura 1).

\section{Función pulmonar}

El porcentaje del $\mathrm{VEF}_{1}$ predicho promedio fue 76,6\% (mínimo: 31\%; máximo 145\%). Treinta y un pacientes $(47,7 \%)$ correspondieron a EPOC leve, 26 pacientes $(40 \%)$ a EPOC moderada y 8 pacientes $(12,3 \%)$ a EPOC severa. El porcentaje del $\mathrm{VEF}_{1}$ promedio en los EPOC leve fue $95,2 \%$, en los moderados $66,8 \%$ y en los severos $36 \%$.

\section{Datos demográficos}

La edad promedio de los pacientes fue 62 años y el 55,4\% fueron mujeres. En los EPOC leves la edad promedio fue 65,2 años, en los moderados 58,5 años y en los severos 60,8 años. En cuanto al género, las mujeres predominaron en los EPOC moderados y severos $(69,3 \%$ y $75 \%$, respectivamente) y los hombres en los EPOC leve (61,3\%).

\section{IMC}

El IMC promedio fue de $28,3 \mathrm{~kg} / \mathrm{m}^{2}$ (mínimo $15,8 \mathrm{~kg} / \mathrm{m}^{2}$; máximo $\left.46,1 \mathrm{~kg} / \mathrm{m}^{2}\right)$. El 36,9\% $(\mathrm{n}=$ 24) fue obeso, $29,2 \%(\mathrm{n}=19)$ sobrepeso, $30,8 \%$ $(\mathrm{n}=20)$ peso normal y $3,1 \%(\mathrm{n}=2)$ bajo peso. En los EPOC leve el IMC promedio fue de 26,9 $\mathrm{kg} / \mathrm{m}^{2}$, en los moderados 29,5 y en los severos 29,9. En los EPOC leve predominaron los pacientes con peso normal $(45,2 \% ; n=14)$ y distribución igual entre los sobrepesos y obesos $(25,8 \%$; $\mathrm{n}=8$ para cada uno). En los EPOC moderado predominaron los obesos $(46,2 \% ; n=12)$ seguido de los pacientes con sobrepeso $(34,6 \% ; n=9)$ $\mathrm{y}$ de peso normal $(15,4 \% ; n=4)$. En los EPOC severos también predominaron los obesos $(50 \%$; $\mathrm{n}=4$ ) seguido de igual porcentaje de sobrepeso y peso normal $(25 \% ; n=2)$ (Figura 2$)$.

\section{Exposición a tabaco y biomasa}

El 78,5\% $(\mathrm{n}=51)$ de los pacientes tenía antecedentes de tabaquismo al momento del ingreso. De ellos, 48 tenían el registro del índice paquete/ año (IPA), el que fue en promedio de 29,8. En los EPOC leve, el 77,4\% $(n=24)$ de los pacientes eran fumadores, con un IPA promedio de 28,6. En los moderados, el $85 \%(\mathrm{n}=17)$ era fumador, con un IPA promedio de 30,8. En los severos, el $87,5 \%(\mathrm{n}=7)$ tenía el antecedente de tabaquismo, con un IPA promedio de 31,7 (Figura 3).

El $81,5 \%$ de los ingresos $(n=53)$ tenía registrado el antecedente de la exposición a biomasa (leña y/o carbón), estando presente en el 73,6\% $(\mathrm{n}=39)$ de ellos. El 50,1\% $(\mathrm{n}=27)$ de los pacientes con registro tenía exposición tanto a
233 pacientes ingresados

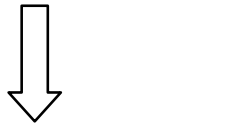

104 con control último año

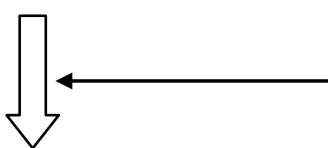

7 sin registro de espirometría 13 sólo datos de espirometría 1 con registro pero sin datos

83 con espirometría de ingreso

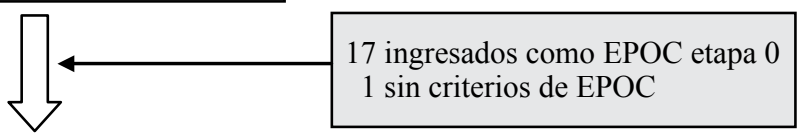

65 con criterios espirométricos de EPOC
Figura 1. Flujograma de inclusión y exclusión de pacientes con EPOC. 


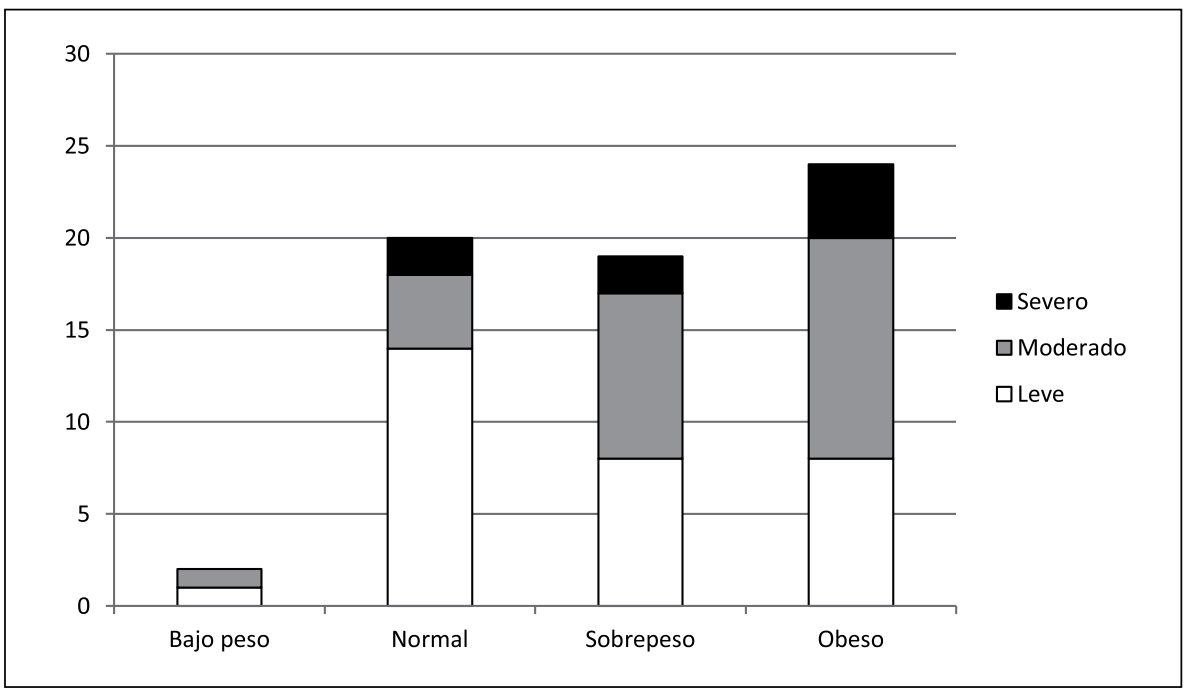

Figura 2. Distribución del índice de masa corporal según clasificación GOLD de la EPOC.

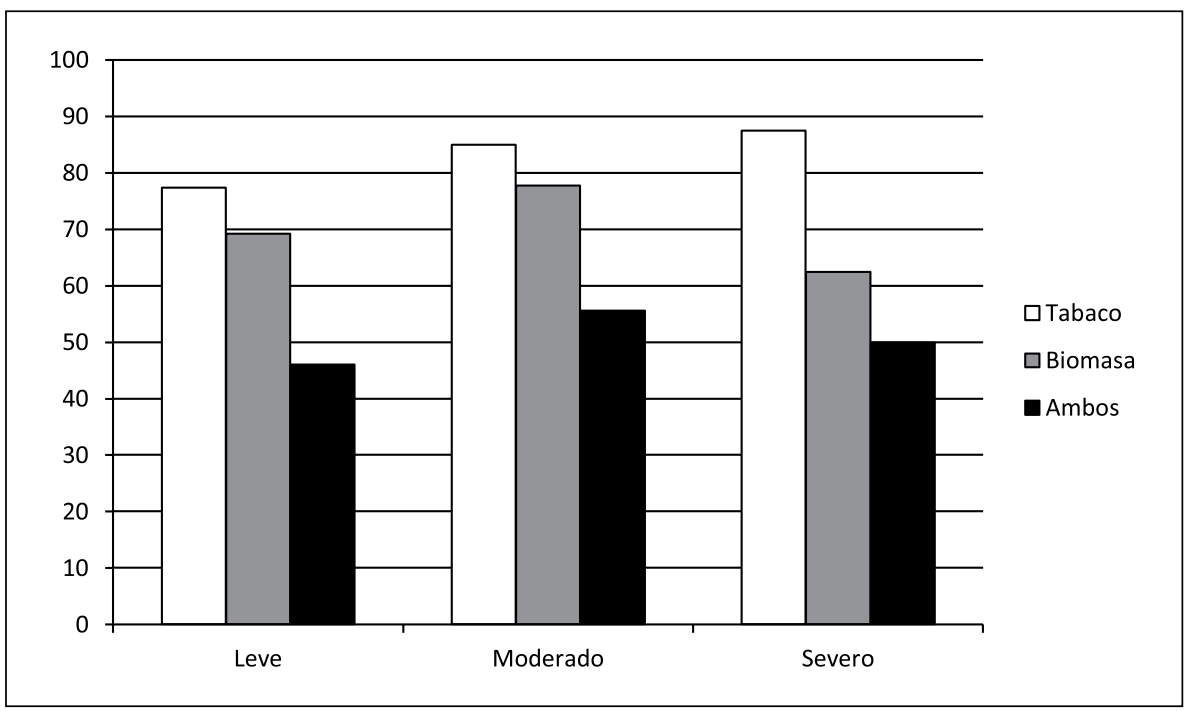

Figura 3. Distribución (en porcentaje) de la exposición a tabaco, biomasa y ambos según grado de severidad de la EPOC.

tabaco como a biomasa. En los EPOC leve, el $69,2 \%(\mathrm{n}=18)$ tenía exposición a biomasa y el $46,1 \%(\mathrm{n}=12)$ tenía a tabaco más biomasa. El 77,8\% $(n=14)$ de los EPOC moderados tenía exposición a biomasa y el 55,6\% $(\mathrm{n}=10)$ a tabaco y biomasa. En los EPOC severos la exposición se observó en el $62,5 \%(\mathrm{n}=5)$ para la biomasa y en el 50\% $(\mathrm{n}=4)$ para biomasa y tabaco (Figura 3).

\section{Radiografía de tórax}

El $80 \%(\mathrm{n}=52)$ de los pacientes tenía una radiografía de tórax al momento del ingreso. El resto $(\mathrm{n}=13)$ no tenía el registro. La mayoría de los pacientes $(\mathrm{n}=30 ; 57,7 \%)$ mostraba cambios imagenológicos sugerentes de atrapamiento aéreo. En el 11,5\% $(n=6)$ las imágenes eran su- gerentes de TBC secuelar y en el 13,5\% $(n=6)$ sugerían enfisema.

\section{Comorbilidades}

El $61,5 \%(n=40)$ de los pacientes tenía alguna comorbilidad al ingreso. De ellos, el 65\% $(\mathrm{n}=26)$ tenía una sola comorbilidad, el 27,5\% $(n=11)$ dos comorbilidades y el 7,5\% $(n=3) 3$ comorbilidades. Las comorbilidades cardiovasculares se detectaron en el $90 \%$ de los pacientes $(\mathrm{n}=36)$, siendo la hipertensión arterial la que más se repitió $(87,5 \% ; \mathrm{n}=35)$ seguido de la diabetes mellitus $(32,5 \% ; n=13)$ (Tabla 1). Los EPOC leves y moderados tuvieron porcentaje similar de pacientes con comorbilidades $(66,6 \%$ y $65,4 \%$, respectivamente) pero los primeros tuvieron mayor porcentaje de pacientes con una 
Tabla 1. Distribución de comorbilidades -según número y tipo- en pacientes con EPOC

\begin{tabular}{|c|c|c|}
\hline & $\mathbf{n}$ & $\%$ \\
\hline Una comorbilidad & 26 & 65 \\
\hline Hipertensión arterial & 21 & \\
\hline Estenosis aórtica & 1 & \\
\hline Artritis reumatoídea & 1 & \\
\hline Daño hepático crónico & 1 & \\
\hline Diabetes mellitus & 1 & \\
\hline Reflujo gastroesofágico & 1 & \\
\hline Dos comorbilidades & 11 & 27,5 \\
\hline Hipertensión arterial + diabetes mellitus & 9 & \\
\hline Hipertensión arterial + arritmia & 1 & \\
\hline Hipertensión arterial + insuficiencia cardíaca & 1 & \\
\hline Tres comorbilidades & 3 & 7,5 \\
\hline Hipertensión arterial + diabetes mellitus + epilepsia & 1 & \\
\hline Hipertensión arterial + diabetes mellitus + infarto agudo del miocardio & 1 & \\
\hline Hipertensión arterial + diabetes mellitus + hipotiroidismo & 1 & \\
\hline
\end{tabular}

Tabla 2. Terapia indicada al momento del ingreso a sala ERA de pacientes con EPOC

\begin{tabular}{|lcc|}
\hline Terapia & n & \% \\
\hline Monoterapia & 27 & 41,5 \\
$\quad$ Broncodilatador de acción corta SOS* & 11 & 16,9 \\
$\quad$ Broncodilatador de acción corta Reg** & 16 & 24,6 \\
\hline Terapia combinada & 33 & 50,7 \\
$\quad$ Broncodilatador de acción corta SOS* + corticoide inhalado & 11 & 16,9 \\
$\quad$ Broncodilatador de acción corta Reg** + corticoide inhalado & 22 & 33,8 \\
\hline Triterapia & 4 & 6,1 \\
$\quad$ Broncodilatador de acción corta SOS* + corticoide inhalado + LABA*** & 1 & 1,5 \\
$\quad$ Broncodilatador de acción corta Reg** + corticoide inhalado + LABA*** & 2 & 3,1 \\
$\quad$ Broncodilatador de acción corta SOS* + broncodilatador de acción corta Reg**+teofilina & 1 & 1,5 \\
\hline Terapia con 4 fármacos & 1 & 1,5 \\
$\quad$ Broncodilatador de acción corta Reg** + corticoide inhalado + teofilina & 1 & 1,5 \\
\hline
\end{tabular}

*En caso de necesidad; **en forma regular; ***agonista $\beta$ adrenérgico de larga acción inhalado.

comorbilidad ( $70 \%$ versus $56,2 \%)$ y los moderados mayor porcentaje de pacientes con dos comorbilidades $(31,3 \%$ versus $25 \%)$.

\section{Exacerbaciones agudas y hospitalizaciones}

Estos datos no se pudieron obtener con certeza ya que en las cartolas de control no está especificado, las fichas electrónicas son posteriores al inicio de la sala ERA y no todas tienen el registro de las crisis pese a existir la opción de este. No hay forma de cruzar datos con las atenciones en los diversos servicios de urgencias.

\section{Tratamiento indicado}

$\mathrm{Al} 41,5 \%$ de los pacientes $(\mathrm{n}=27)$ se les indicó monoterapia con un broncodilatador de acción corta $(\mathrm{BDc})$ ya sea en forma regular $(24,6 \% ; n=$
16) o en caso de necesidad $(16,9 \% ; \mathrm{n}=11)$. Al $50,7 \%(\mathrm{n}=33)$ se les indicó terapia combinada con BDc más corticoide inhalado (CDi). Al $6,15 \%(n=4)$ se les indicó triterapia (2 pacientes con $\mathrm{BDc}$ más $\mathrm{CDi}$ más $\mathrm{LABA}$; uno con $\mathrm{BDc}$ en forma regular más $\mathrm{BDc}$ en caso de necesidad más $\mathrm{CDi}$; uno con $\mathrm{BDc}$ en forma regular más $\mathrm{BDc}$ en caso de necesidad más teofilina). Un paciente $(1,5 \%)$ recibió terapia con cuatro drogas (BDc más LABA más CDi más teofilina) (Tabla 2).

$\mathrm{Al}$ analizar la indicación del tratamiento según grado de severidad vemos que la monoterapia con BDc fue indicada al 61,3\% $(\mathrm{n}=19)$ de los pacientes con EPOC leve, al 23,1\% $(n=6)$ de los EPOC moderados y al $12,5 \%(\mathrm{n}=1)$ de los severos. La terapia combinada (BDc más $\mathrm{CDi}$ ) fue indicada al 38,7\% $(\mathrm{n}=12)$ de los EPOC leves; 


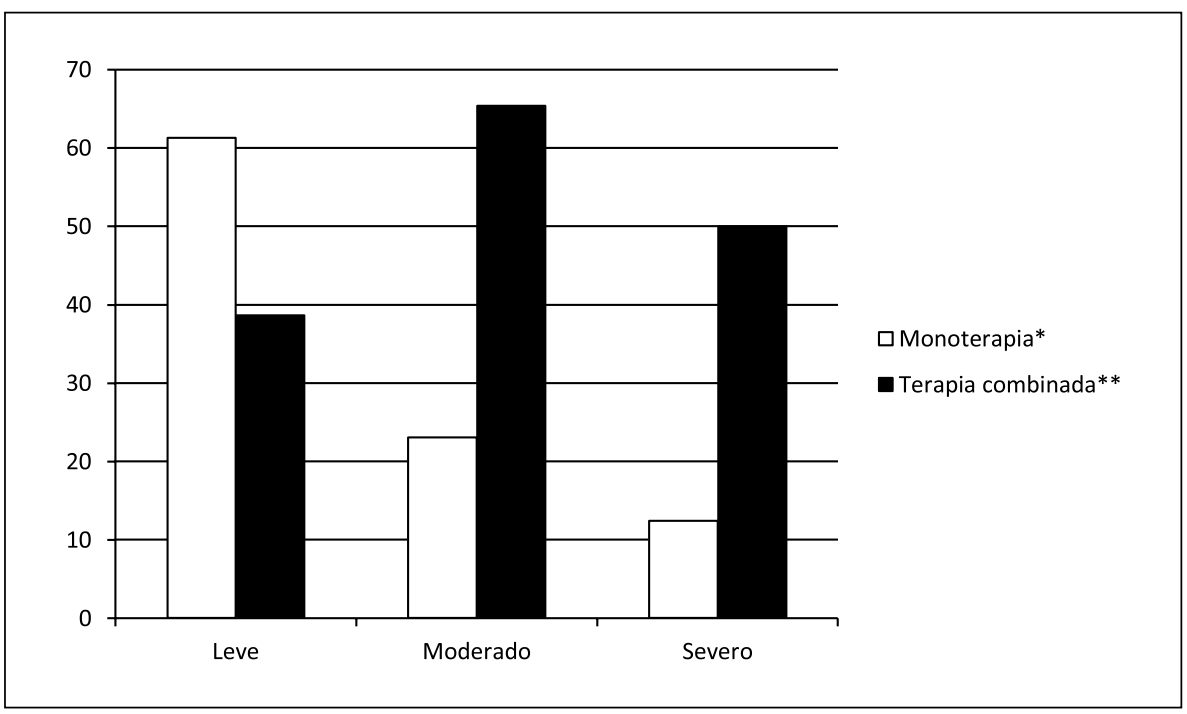

Figura 4. Distribución (en porcentaje) del número de fármacos indicados según severidad de la EPOC. *Incluye broncodilatadores de acción corta; **Incluye broncodilatadores de acción corta más corticoides inhalados.
$65,4 \%(\mathrm{n}=17)$ de los moderados y $50 \%(\mathrm{n}=4)$ de los severos (Figura 4).

\section{Discusión}

Este estudio descriptivo muestra que el perfil clínico al ingreso de los pacientes EPOC que se controlan en la sala ERA en un CESFAM de la comuna de Puente Alto y que mantienen sus controles activos se caracteriza por ser predominantemente adultos mayores de 60 años, mujeres y con distribución similar de leves y moderados según compromiso funcional espirométrico. La mayoría tiene exposición a tabaco y biomasa (la mitad con exposición a ambos) y presentan comorbilidades, preferentemente cardiovasculares.

La gran cantidad de pacientes sin controles activos del programa $(n=129)$ corresponden en su mayoría a fallecidos $(n=44)$ y a traslados, tanto a otras comunas como al nivel secundario $(n=50)$. Hubo 4 pacientes que salieron del programa (un alta y 3 cambios de diagnóstico) y 5 reingresaron, por lo que tenían doble cartola de control y por ende no fueron contabilizados en el total de la muestra. El resto $(n=31)$ eran pacientes que no se habían presentado al control, a pesar que 14 de ellos habían recibido "citación por terreno". Creemos que la incapacidad para "rescatar" a estos pacientes se debe principalmente a la falta de horas efectivas de los integrantes dedicados al programa, ya que una parte de las horas asignadas a la Sala ERA son "asignadas" para apoyar el cuplimiento de otros objetivos sanitarios de la Atención Primaria de Salud.
De los pacientes EPOC que se mantenían con controles activos, 18 fueron ingresados al programa teniendo espirometría normal. Si bien estos pacientes no cumplen los criterios espirométricos actuales de EPOC, debemos recordar que hasta el año 2006 se recomendaba ingresar al programa a los llamados "EPOC etapa 0", es decir, a aquellos sujetos con espirometría normal, presencia de síntomas respiratorios y exposición a factores de riesgo de $\mathrm{EPOC}^{17}$. Es en este contexto en el que fueron ingresados estos pacientes, pero como esta recomendación se abandonó desde el año 2006 en adelante ${ }^{18}$, no los incluimos en los análisis. Es interesante mencionar que sólo 4 de ellos evolucionaron en el tiempo con una obstrucción espirométrica (datos no incluidos).

Como las salas ERA están pensadas para la atención primaria, no es sorprendente que en nuestro estudio predominaran los pacientes con EPOC leves y moderados. Debido a que el número de casos con EPOC severo fue muy escaso (8 pacientes) y a que las guías clínicas nacionales ${ }^{12}$ recomiendan que estos pacientes se deberían controlar en el nivel secundario, no los consideraremos en esta discusión. Sólo podemos mencionar que en el caso de nuestros pacientes severos, se mantienen en control tanto en este CESFAM como en el Hospital base por motivos administrativos (retiro de medicamentos) y para el manejo rápido de las exacerbaciones.

La edad avanzada en nuestra muestra de pacientes se condice con la mayoría de los estudios ${ }^{19,20,21}$. Esto sugiere que el diagnóstico se está haciendo en forma tardía, por lo que sería necesario reforzar la búsqueda dirigida de esta 


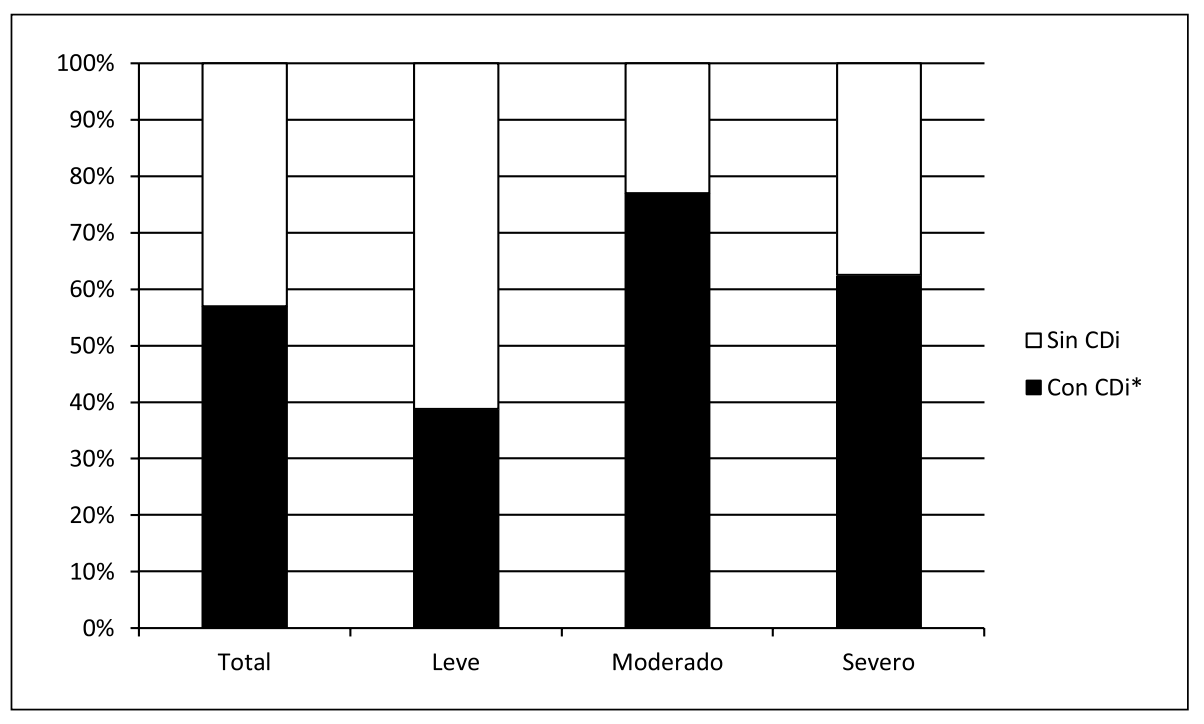

Figura 5. Porcentaje de pacientes con y sin corticoides indicados según severidad de la EPOC. *: Corticoides inhalados.

patología en las consultas de morbilidad general.

En nuestra cohorte de pacientes predominaron levemente las mujeres, especialmente en los EPOC moderados. Esta distribución no es la que muestra la mayoría de los estudios de EPOC a nivel mundial ${ }^{6,22}$. Este hallazgo necesita mayor investigación a futuro.

Existen estudios que han mostrado que el bajo peso se asocia con mayor mortalidad en los pacientes con EPOC ${ }^{23}$. Nuestro pacientes con EPOC tenían predominantemente sobrepeso, lo que concuerda con los resultados del estudio PLATINO, el que encontró que en los pacientes EPOC de Santiago predominaban también aquellos con sobrepeso $^{24}$. Al hacer el análisis según clasificación GOLD, vemos que en los leves predominaron los pacientes con peso normal y en los moderados los obesos. Esto podría tener significancia clínica ya que hay datos que sugieren que la obesidad se asociaría con mayor inflamación sistémica y, por tanto, con mayor morbimortalidad cardiovascular en estos pacientes ${ }^{25}$.

Como era de esperarse, la gran mayoría de los pacientes tenía exposición a tabaco, pero no hubo mayor diferencia entre los EPOC leves y moderados. El IPA fue en general más alto que en otros estudios mucho más grandes ${ }^{26}$, hallazgo que necesita mayor investigación. Llama la atención en nuestros pacientes la gran exposición al humo de combustión de leña y/o carbón, la que llega a ser casi tan frecuente como la exposición al tabaco, siendo superior a lo publicado en otros estudios $^{6}$. Existen datos internacionales que muestran que las mujeres están más expuestas al humo de biomasa y que el grado de severidad de la obstrucción bronquial es menor que en los expuestos al tabaco ${ }^{27}$. En nuestro estudio no hubo diferencias entre los hombres y las mujeres en cuanto a la exposición al humo de biomasa y los EPOC moderados presentaron mayor porcentaje de exposición tanto a biomasa como a la combinación de tabaco y biomasa. Si bien nuestros datos no permiten hacer una estimación exacta de la cantidad de exposición a la combustión de biomasa ni tenemos cómo compararla a grado de exposición de la población general de la comuna de Puente Alto, creemos que es un dato interesante de profundizar debido a las implicancias tanto clínicas como terapéuticas que podrían existir.

La presencia de comorbilidades en la EPOC impacta en la calidad de vida de los pacientes ${ }^{28,29}$ y han sido consideradas como causas de mortalidad primaria en más del $60 \%$ de los casos en grandes estudios ${ }^{30}$. En nuestra cohorte, un porcentaje importante de los pacientes $(61,5 \%)$ tenía al menos una comorbilidad al momento del ingreso y cerca de un cuarto tenía dos comorbilidades. La hipertensión arterial fue la comorbilidad que predominó ampliamente estando presente en el $87,5 \%$, lo que está acorde con los grandes estudios publicados ${ }^{31}$. Hubo un leve mayor porcentaje de pacientes con dos comorbilidades en el grupo con EPOC moderado versus el leve.

El tratamiento indicado, en un número importante de pacientes, no se ajustó a las recomendaciones nacionales ni internacionales, existiendo un uso excesivo de corticoides inhalados. Cerca del $57 \%$ de todos los pacientes recibieron corticoides inhalados $(38,7 \%$ de los EPOC leve y 76,9\% de los moderados) (Figura 5). Esta 
tendencia ha sido identificada también en otros estudios $^{32-35}$. En nuestra Sala ERA, creemos que la falta de un programa de capacitación y educación médica contínua es la principal causa de prescripción inadecuada de corticoides inhalados.

Creemos que nuestros resultados podrían identificar ciertas características interesantes de nuestra población EPOC que difieren en algunos aspectos y agregan otros a los escasos datos que conocemos de los pacientes EPOC en Chile. El estudio PLATINO ${ }^{2}$ fue un estudio que investigó la prevalencia de la EPOC en cinco ciudades latinoamericanas, incluyendo Santiago de Chile y es, hasta donde nosotros conocemos, la única fuente de datos de algunas características clínicas de estos pacientes que existe de nuestro país. En dicho estudio la prevalencia de la EPOC fue mayor en hombres, mayores de 60 años, con sobrepeso y con un IPA $>20$. Si bien la población analizada en este estudio y el nuestro difieren, ya que en PLATINO se evaluó una muestra de la población general y nuestro estudio estuvo conformado por pacientes con diagnóstico ya establecido de EPOC, nuestros resultados concordaron con la mayoría de las características reportadas en el estudio PLATINO, excepto que nuestros pacientes fueron principalmente mujeres. La explicación más probable de esta diferencia es justamente el tipo de población estudiada. Nuestro estudio agrega, además, características no investigadas en el estudio PLATINO como la alta exposición a biomasa y la gran frecuencia de comorbilidades cardiovasculares.

Este estudio tiene varias limitaciones. Primero, el diseño retrospectivo observacional, el que tiene per se un sinnúmero de limitaciones ampliamente reconocidas. Pero a pesar de esto, tiene también las ventajas de ser un estudio hecho en el "contexto de vida real" y, a nuestro entender, es el primero realizado en nuestro país. Segundo, la escasa población analizada, lo que no permite generalizar los hallazgos. Pero esta es la población en control en este CESFAM, por lo que estos datos pueden ayudar al manejo programático de esta sala ERA. Tercero, existen muchos problemas en el registro de los datos, lo que imposibilita tener toda la información necesaria en todos los pacientes. Esto se hace evidente principalmente en lo que dice relación con las exacerbaciones agudas, y la falta de este dato impide hacer una evaluación completa, tomar decisiones terapéuticas más efectivas y realizar un seguimiento adecuado de los pacientes.

En suma, a pesar de estas limitaciones, nuestro estudio nos permitió identificar algunas características interesantes en los pacientes EPOC que se controlan en la sala ERA del CESFAM Cardenal Raúl Silva Henríquez de la comuna de Puente Alto: (a) son preferentemente mujeres mayores de 60 años con alta exposición tanto a tabaco como a humo de combustión de biomasa y alta presencia de comorbilidades, especialmente la hipertensión arterial; (b) como en la mayoría de los estudios, el tratamiento no se ajustó a las recomendaciones nacionales, especialmente en lo que dice relación con la indicación de corticoides inhalados.

Creemos que sería importante replicar este estudio en las otras salas ERA tanto de esta comuna como en el resto de la Región Metropolitana e idealmente en todas las comunas del país para evaluar si estas características clínicas se repiten o si aparecen otras, con el fin de optimizar las estrategias de prevención, diagnóstico y manejo de la EPOC en Chile.

\section{Bibliografía}

1.- LOZANO R, NAGHAVI M, FOREMAN K, LIM S, SHIBUYA K, ABOYANS V, ABRAHAM J, et al. Global and regional mortality from 235 causes of death for 20 age groups in 1990 and 2010: a systematic analysis for the Global Burden of Disease Study 2010. Lancet 2012; 380: 2095-128.

2.- MENEZES A M, PÉREZ-PADILLA R, JARDIM J R B, MUIÑO A, LÓPEZ M V, VALDIVIA G, et al, PLATINO Team: Chronic obstructive pulmonary disease in five Latin American cities (the PLATINO study): a prevalence study. Lancet 2005; 366: 1875-81.

3.- VESTBO J, AGUSTI A, WOUTERS E F M, BAKKE $\mathrm{P}$, CALVERLEY P M A, CELLI B, COXSON H, et al. On behalf of the Evaluation of COPD Longitudinally to Identify Predictive Surrogate Endpoints Study Investigators. Should We View Chronic Obstructive Pulmonary Disease Differently after ECLIPSE? A Clinical Perspective from the Study Team. Am J Respir Crit Care Med 214; 189: 1022-30.

4.- CERVERI I, ACCORDINI S, VERLATO G, CORSICO A, ZOIA M C, CASALI L, BURNEY P, DE MARCO R, for the European Community Respiratory Health Survey (ECRHS) Study Group. Variations in the prevalence across countries of chronic bronchitis and smoking habits in young adults. Eur Respir J 2001; 18 : 85-92.

5.- BUIST S, MCBURNIE M A, VOLLMER W M, GILLESPIE S, BURNEY P, MANNINO D M, et al. BOLD Collaborative Research Group. International variation in the prevalence of COPD. (The BOLD Study): a population-based prevalence study. Lancet 2007; 370 : 741-50.

6.- MIRAVITLLES M, MURIO C, TIRADO-CONDE G, LEVY G, MUELLEROVA H, SORIANO J B, RAMÍ- 
REZ-VENEGAS A, et al. Geographic differences in clinical characteristics and management of COPD: the EPOCA study. International Journal of COPD 2008; 3 : 803-14.

7.- SOBRADILLO PEÑA V, MIRAVITLLES M, GABRIEL R, JIMÉNEZ-RUIZ C A, VILLASANTE C, MASA J F, et al. Geographic Variations in Prevalence and Underdiagnosis of COPD. Results of the IBERPOC Multicentre Epidemiological Study. Chest 2000; 118: 981-9.

8.- MONTES DE OCA M, HALBERT R J, LÓPEZ M V, PÉREZ-PADILLA R, TÁLAMO C, MORENO D, et al. The chronic bronchitis phenotype in subjects with and without COPD: the PLATINO study. Eur Respir J 2012; 40: $28-36$

9.- HERLAND K, AKSELSEN J P, SKJØNSBERG O H, BJERMER L. How representative are clinical study patients with asthma or COPD for a larger "real life", population of patients with obstructive lung disease? Respir Med 2005; 99: 11-9.

10.- VIEJO-BAÑUELOS J L, PUEYO-BASTIDA A, FUEYO-RODRÍGUEZ A. Characteristics of outpatients with COPD in daily practice: The E4 Spanish project. Respir Med 2006; 100: 2137-43.

11.- Gobierno de Chile. Ministerio de Salud. Programa de Control de las Enfermedades Respiratorias del Adulto en Chile.

12.- Ministerio de Salud. Guía Clínica Enfermedad Pulmonar Obstructiva Crónica de tratamiento ambulatorio. Santiago: MINSAL 2013.

13.- http://web.minsal.cl/portal/url/item/e03c08fac00143dee0400101650176c1.pdf

14.- GUTIÉRREZ M, BEROÍZA T, BORZONE G, CAVIEDES I, CÉSPEDES J, MORENO R, et al. Espirometría: Manual de procedimientos. Sociedad Chilena de Enfermedades Respiratorias 2006. Rev Chil Enf Respir 2007; 23: $31-42$

15.- World Health Organization. Obesity: preventing and managing the global epidemic. Report of a WHO consultation. World Health Organization technical report series, $\mathrm{n}^{\circ} 894,2000$.

16.- Global Initiative for Chronic Obstructive Pulmonary Disease. Global Strategy for the Diagnosis, Management, and Prevention of Chronic Obstructive Pulmonary Disease. Updated 2011. Disponible en: http://www.goldcopd.org/uploads/user/files/GOLD_Report_2011_Feb21. pdf. Consultada el 27 de marzo de 2015.

17.- Global Initiative for Chronic Obstructive Pulmonary Disease. Global Strategy for the Diagnosis, Management, and Prevention of Chronic Obstructive Pulmonary Disease. Revised 2004. Disponible en: http://www.goldcopd.org/uploads/user/files/GOLDWkshp2004Clean. pdf. Consultada el 27 de marzo de 2015.

18.- Global Initiative for Chronic Obstructive Pulmonary Disease. Global Strategy for the Diagnosis, Management, and Prevention of Chronic Obstructive Pulmonary
Disease. Revised 2006. Disponible en: http://www.goldcopd.org/uploads/user/files/GOLDReport2006_0122. pdf. Consultada el 27 de marzo de 2015.

19.- CALVERLEY P M A, ANDERSON J A, CELLI B, FERGUSON G T, JENKINS C, JONES P W, et al, for the TORCH investigators. Salmeterol and Fluticasone Propionate and Survival in Chronic Obstructive Pulmonary Disease. N Engl J Med 2007; 356: 775-89.

20.- TASHKIN D P, CELLI B, SENN S, BURKHART D, KESTEN S, MENJOGE S, et al, for the UPLIFT Study Investigators. A 4-Year Trial of Tiotropium in Chronic Obstructive Pulmonary Disease. N Engl J Med 2008; 359: $1543-54$

21.- VESTBO J, EDWARDS L D, SCANLON P D, YATES $\mathrm{J}$ C, AGUSTI A, BAKKE P, et al, for the ECLIPSE Investigators. Changes in Forced Expiratory Volume in 1 Second over Time in COPD. N Engl J Med 2011; 365: 1184-92.

22.- LÓPEZ VARELA M V, MONTES DE OCA M, HALBERT R J, MUIÑO A, PÉREZ-PADILLA R, TÁLAMO C, et al, for the PLATINO Team. Sex-related differences in COPD in five Latin American cities: the PLATINO study. Eur Respir J 2010; 36: 1034-41.

23.- LANDBO C, PRESCOTT E, LANGE P, VESTBO J, ALMDAL T P. Prognostic Value of Nutritional Status in Chronic Obstructive Pulmonary Disease. Am J Respir Crit Care Med 1999; 160: 1856-61.

24.- MONTES DE OCA M, TÁLAMO C, PÉREZ-PADILLA R, JARDIM J R B, MUIÑO A, LÓPEZ M V, et al, for the PLATINO Team. Chronic obstructive pulmonary disease and body mass index in five Latin America cities: The PLATINO study. Respir Med 2008; 102: 642-50.

25.- AGUSTÍ A, EDWARDS L D, RENNARD S I, MACNEE W, TAL-SINGER R, MILLER B E, et al, for the Evaluation of COPD Longitudinally to Identify Predictive Surrogate Endpoints (ECLIPSE) Investigators. Persistent Systemic Inflammation is Associated with Poor Clinical Outcomes in COPD: A Novel Phenotype. PLoS One 2012; 7: e37483.

26.- BLANC P D, MENEZES A M B, PLANA E, MANNINO D M, HALLAL P C, TOREN K, et al. Occupational exposures and COPD: an ecological analysis of international data. Eur Respir J 2009; 33: 298-304.

27.- RAMÍREZ-VENEGAS A, SANSORES R H, PÉREZPADILLA R, REGALADO J, VELÁZQUEZ A, SÁNCHEZ C, et al. Survival of Patients with Chronic Obstructive Pulmonary Disease Due to Biomass Smoke and Tobacco. Am J Respir Crit Care Med 2006; 173: 393-7.

28.- CRISAFULLI E, COSTI S, LUPPI F, CIRELLI G, CILIONE C, COLETTI O, et al. Role of comorbidities in a cohort of patients with COPD undergoing pulmonary rehabilitation. Thorax 2008; 63: 487-92.

29.- VAN MANEN J G, BINDELS P J E, DEKKER F W, BOTTEMA B J A M, VAN DER ZEE J S, IJZER- 
MANS C J, et al. The influence of COPD on healthrelated quality of life independent of the influence of comorbidity. J Clin Epidemiol 2003; 56: 1177-84.

30.- MCGARVEY L P, JOHN M, ANDERSON J A, ZVARICH M, WISE R A. Ascertainment of cause-specific mortality in COPD: operations of the TORCH Clinical Endpoint Committee. Thorax 2007; 62: 411-5.

31.- DIVO M, COTE C, DE TORRES J P, CASANOVA C, MARÍN J M, PINTO-PLATA V, et al, for the BODE Collaborative Group. Comorbidities and Risk of Mortality in Patients with Chronic Obstructive Pulmonary Disease. Am J Respir Crit Care Med 2012; 186: 155 61.

32.- LÓPEZ VARELA M V, MUIÑO A, PÉREZ PADILLA R, JARDIM J R, TÁLAMO C, MONTES DE OCA M, et al y Grupo PLATINO. Tratamiento de la EPOC en 5 ciudades de América Latina: estudio PLATINO. Arch Bronconeumol 2008; 44: 58-64.

33.- MIRAVITLLES M, DE LA ROZA C, NABERAN K, LAMBAN M, GOBARTT E, MARTIN A. Use of spirometry and patterns of prescribing in COPD in primary care. Respir Med 2007; 101: 1753-60.

34.- ROCHE N, LEPAGE T, BOURCEREAU J, TERRIOUX P. Guidelines versus clinical practice in the tratment of chronic obstructive pulmonary disease. Eur Respir J 2001; 18: 903-8.

35.- DE MIGUEL J, IZQUIERDO ALONSO J L, RODRÍGUEZ GONZÁLEZ-MORO, DE LUCAS RAMOS P, MOLINA PARIS J. Tratamiento farmacológico de la EPOC en dos niveles asistenciales. Grado de adecuación a las normativas recomendadas. Arch Bronconeumol 2003; 39: 195-202.

Correspondencia a:

Gonzalo Alvear T.

Clínica Miguel de Servet

Providencia

Email: gonzaloalvear@yahoo.com 